\title{
DYNAMIC CHARACTERIZATION OF IN-PLANE BULK ACOUSTIC RESONATORS USING HIGH-SENSITIVITY OPTICAL REFLECTION MEASUREMENTS \\ Vikrant J. Gokhale ${ }^{1,2}$ and Jason J. Gorman ${ }^{1}$ \\ ${ }^{1}$ National Institute of Standards and Technology, Gaithersburg, MD 20899-8212, USA \\ ${ }^{2}$ University of Michigan, Ann Arbor, MI 48109-2122, USA
}

\begin{abstract}
Two experimental techniques for measuring dynamic displacement and strain for in-plane bulk acoustic resonators are presented. These techniques, optical knife-edge and photoelastic measurements, can characterize in-plane high-frequency vibrations with a degree of precision and simplicity that has not been shown previously with MEMS. The measurements are spatially resolved and can be used to reconstruct the vibrational mode shape of the resonator. Experimental results presented here are acquired using both methods on a single crystal silicon bulk acoustic resonator (SiBAR) with a fundamental resonance frequency of $\approx 13.6 \mathrm{MHz}$.
\end{abstract}

\section{INTRODUCTION}

Capacitive [1] and piezoelectric [2] bulk acoustic resonators (BARs) have received considerable attention over the last decade due to applications in RF timing, filtering, and sensing. Much of the success of these resonators stems from their ability to vibrate in a specific mode with a high mechanical quality factor and hence a sharp spectral linewidth. Contemporary design of micromechanical resonators is heavily reliant on analytical equations and finite element analysis (FEA) of the device, followed by electrical validation. However, the drawback of purely electrical characterization is that it presents only an aggregate signal at the output transduction port of the system (e.g., capacitively induced current). As a result, all-electrical transduction obscures the internal mechanics of the resonator, making it difficult to determine the causes of spurious modes, nonlinearities, and other phenomena. In contrast, optical techniques can provide more detailed and precise characterization of the resonator through spatially and temporally resolved measurement of the absolute device displacement. Laser Doppler vibrometry has been used successfully to measure BARs but typically only for out-of-plane motion [3]. Recently, three-dimensional laser Doppler vibrometry has been demonstrated but at considerable expense and complexity and with limited bandwidth for BARs [4]. Optical knife-edge [5, 6] and photoelastic [7] measurement techniques provide highsensitivity in-plane motion measurements with simplicity and high bandwidth, as demonstrated by others for surface acoustic waves [7], but have received little attention for BARs, which has motivated the presented research.

\section{DESCRIPTION OF DEVICE \& FABRICATION}

The devices used in this work are silicon bulk acoustic resonators (SiBAR) that vibrate in their extensional in-plane modes (Fig. 1). The SiBARs are fabricated using standard silicon-oninsulator (SOI) processes. First, metal electrodes are patterned and evaporated using liftoff on an SOI wafer. The device layer of the SOI wafer, which is approximately $10 \mu \mathrm{m}$ thick, is patterned and etched using deep reactive ion etching (DRIE) to form the transduction gaps and release etch holes. A transduction gap of $\approx 480 \mathrm{~nm}$ is achieved using $5 \mathrm{X}$ reduction stepper lithography. The DRIE is optimized to provide vertical sidewalls with scallop depths of $\approx 80 \mathrm{~nm} /$ cycle and scalloping undercut on the order of $\approx 15 \mathrm{~nm}$. The wafer is diced into individual chips and the mechanical structure is released by etching the $2 \mu \mathrm{m}$ thick buried oxide layer using hydrofluoric acid vapor.

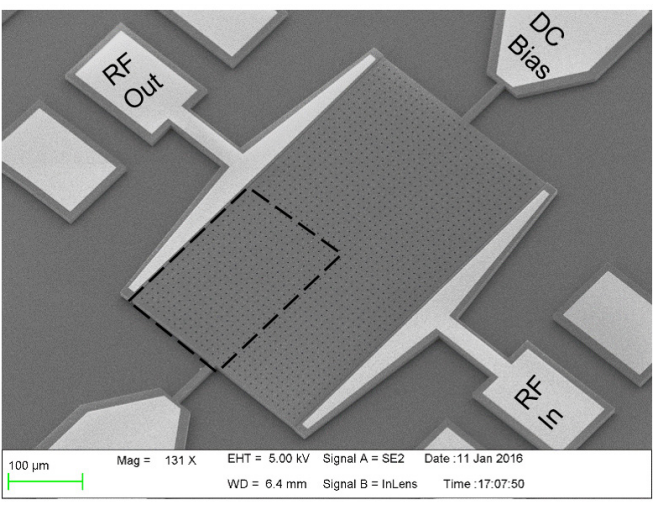

Figure 1: Scanning electron image of a width-extensional single crystal SiBAR with a transduction gap of $480 \mathrm{~nm}$ and a measured fundamental resonance frequency of $\approx 13.6 \mathrm{MHz}$. The resonator width is $300 \mu \mathrm{m}$, with an expected resonance frequency of 13.8 MHz. These SiBARs are used as a platform for demonstrating precise dynamic measurements of driven motion and energy dissipation mechanisms. Due to the symmetry of the SiBAR, only one quadrant of the device (boxed) is measured and simulated.

\section{CHARACTERIZATION TECHNIQUES}

\section{Electrical Measurements}

The released SiBAR was first tested electrically using a vector network analyzer (VNA) and ground-signal-ground (GSG) probes. Standard short-open-load-through (SOLT) calibration is performed prior to acquiring the data seen in Fig. 2. It is observed that the device requires a fairly large DC bias to transduce a measurable resonance, and the signal-to-noise ratio (SNR) is low due to the presence of parasitic feedthrough. The effect of parasitics can be reduced by optimized design, a smaller transduction gap, or electronic signal amplification, but as will be demonstrated in further sections, these improvements are not necessary for optical readout and characterization.



(a) Frequency $(\mathrm{MHz})$

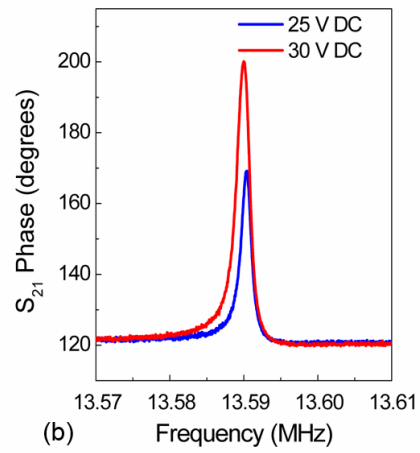

Figure 2: Standard electrical testing of the $\approx 13.6 \mathrm{MHz}$ SiBAR in air, prior to wire-bonding, using GSG probes and a VNA. Magnitude (a) and phase (b) information are shown. Amplification was not used in these tests. No response is seen below $\approx 20 \mathrm{VDC}$ bias, primarily due to the effect of the parasitic feedthrough from the device, bond pads, and handle wafer.

Solid-State Sensors, Actuators and Microsystems Workshop Hilton Head Island, South Carolina, June 5-9, 2016 


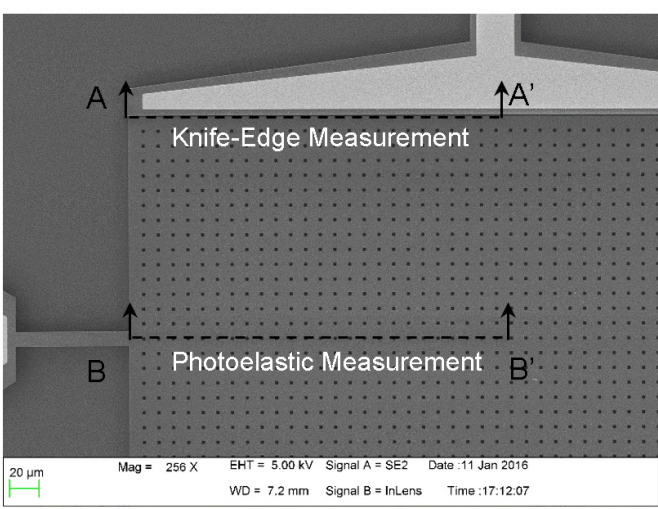

Figure 3: Optical knife-edge measurements are acquired on the free edge (AA') and photoelastic measurements are acquired on the line of maximum strain: the major axis of the device ( $B B$ ').

\section{Optical Experiment Setup}

The devices are mounted on a printed circuit board (PCB) and wire-bonded. The PCB is mounted on a positioning stage with $10 \mathrm{~nm}$ positioning precision along all three linear axes. The SiBAR is actuated by an RF signal from the VNA and a DC bias applied to the body of the resonator. An intensity stabilized helium-neon laser is used as the optical probe $(\lambda=632.8 \mathrm{~nm})$. The laser is focused on the resonator surface using a $20 \mathrm{X}$ objective mounted on a tube microscope, resulting in a Gaussian spot on the device with a diameter of $\approx 2 \mu \mathrm{m}$. The reflected optical signal is detected using a Si PIN photodetector with half-power bandwidth of $200 \mathrm{MHz}$ and a gain of $2000 \mathrm{~V} / \mathrm{W}$ at $633 \mathrm{~nm}$. The photodetector output is fed back to the VNA to provide an output signal relative to the input $\mathrm{RF}$ drive signal. The laser output is attenuated using a polarizer to maximize the signal from the photodetector while limiting the local heating due to the photothermal effect. Precise targeting of the laser spot is achieved by viewing the system in real time with a CCD camera on the microscope, and by monitoring the average output power reflected from the resonator using an oscilloscope. The same experimental setup is used for both optical methods and no recalibration or modification is necessary.

\section{Knife-Edge Measurements}

Optical knife-edge techniques have been used in the past to measure flexural and bulk acoustic resonators [5, 6]. This technique has the potential to measure displacements on the order of $1 \mathrm{pm} / \sqrt{\mathrm{Hz}}$ [6] and below. The measurements are performed by positioning a focused laser spot on any edge of the resonator surface that has high in-plane displacement. The motion of the edge modulates the reflected optical power, leading to a strong signal at the motion frequency. The leading edge of the SiBAR (AA' from Fig. 3) has minimal strain and maximum displacement along the actuation axis. The laser spot is optimally positioned at the point of maximum displacement sensitivity by scanning the spot across the gap and monitoring the photodetector output. As the leading edge of the resonator is driven to move in plane, the change in reflected power is determined by the displacement of the edge. Fig. 4 shows the magnitude and phase of the optical knifeedge signal from the resonator. The inset illustrates the knife-edge scheme and the positioning of the laser spot. Unlike electrical characterization, this method can be used to extract the absolute displacement of every point on the leading edge of the resonator by scanning the laser over the entire edge. In general, this technique can be extended to measure planar displacement on any edge with optical contrast, such as etch holes and electrodes on a piezoelectric resonator.



Figure 4: Reflected knife-edge signal when the laser is focused on the leading edge of the resonator (i.e., point of highest displacement and zero strain). A clear signal is acquired (both magnitude and phase), with an SNR better than $30 \mathrm{~dB}$. The resonator was actuated with $10 \mathrm{~mW} R F$ power and $10 \mathrm{VDC}$ bias in vacuum at $1.33 \mathrm{mPa}$ (10 $\mu$ Torr). Inset: Diagram showing knifeedge measurement with position of the laser spot (not to scale).

Measurements can be acquired on edges of any orientation, allowing displacement measurements along any planar vector. Thus the vector displacements of many points on the resonator can be measured to reconstruct the device dynamics. The measurement SNR is a function of the optical contrast between the resonator and the free space gap. For this SiBAR, the contrast is between the resonator body and the transduction trench (480 nm wide, $12 \mu \mathrm{m}$ deep). For the current measurement, an SNR of $\approx 15 \mathrm{~dB}$ is achieved at DC bias levels as low as $1 \mathrm{~V}$, and up to $40 \mathrm{~dB}$ has been measured at higher voltages. The optical contrast can be improved by removing the substrate under the trench, or by using materials with dissimilar reflectivities for the resonator and the substrate.

\section{Photoelastic Strain Measurements}

Another optical method for characterizing the dynamics of high frequency resonators is to utilize the photoelastic effect, in which the index of refraction for the resonator material is modulated by strain-inducing acoustic waves. This technique has been used in the past to measure the propagation of surface acoustic waves [7]. As the resonator undergoes periodic in-plane strain, the refractive index of the material changes at the same rate, which in turn modulates the reflected intensity of the laser spot. The change in refractive index as a function of the dynamic strain is given by $\Delta n=-\left(0.5 n^{3} p_{i j}\right) \Delta \varepsilon_{i j}$, where $n$ is the nominal refractive index, $p_{i j}$ is the set of photoelastic coefficients for the material, and $\Delta \varepsilon_{i j}$ is the change in strain [7]. Most significantly, this technique does not require an edge with optical contrast and can be used on unpatterned surfaces. A single material with uniform optical properties and a clean flat surface is the ideal platform for performing photoelastic measurements. The advantage of using a single crystal silicon device such as the SiBAR is that the photoelastic coefficients of the material are well known, thus enabling the extraction of absolute planar strain at any point on the surface. The largest surface strain in the resonator is located along the central line (BB' from Fig. 3). Representative amplitude and phase data are shown in Fig. 5. As with knife-edge measurements, the photoelastic measurements can be taken at multiple points on the surface in order to generate a composite vector plot of the surface strain dynamics of the resonator. 


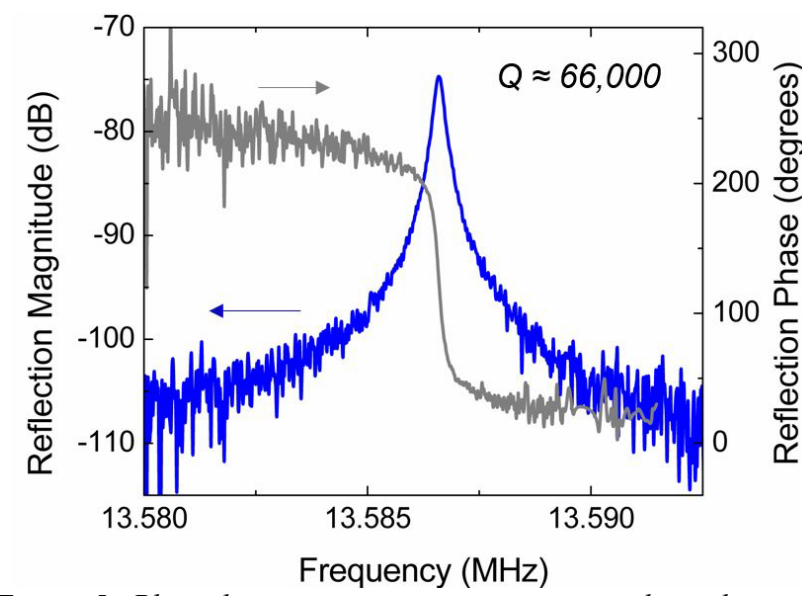

Figure 5: Photoelastic measurement at a point along the major axis of the resonator. It is clear that there is strong intensity modulation at the resonance frequency. Both magnitude and phase information are shown, with an $S N R$ of $\approx 25 \mathrm{~dB}$ for the magnitude. Same test conditions as in Fig. 4.

\section{Mode Shape Identification}

A major advantage of using these two optical techniques is that they provide spatially-resolved measurements across the entire resonator, thereby enabling reconstruction of the vibration mode shapes. The fundamental mode shape along the leading edge of the SiBAR was measured with the knife-edge technique and the strain profile along the center axis of the SiBAR was measured with the photoelastic technique. These results are compared with those from FEA simulations in Fig. 6. While there is some qualitative agreement between the expected and measured mode shape and strain profile, the differences are large enough to indicate that either the measurement, the simulation, or both are inaccurate. Due to the simplicity and repeatability of the optical measurements, we believe that the FEA model is the less accurate of the two due to unmodeled fabrication imperfections and the meshing complexity of the etch holes. This assertion is supported by our observations of mode suppression and a significant loss of transduction efficiency that were not predicted by the FEA simulations.


(b)

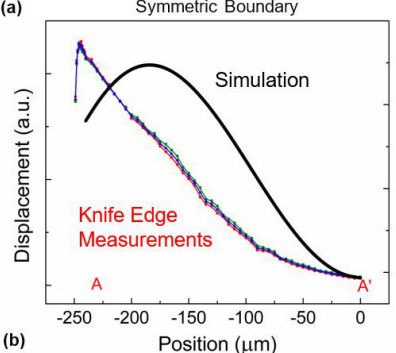

Figure 6: Fundamental mode shape and dynamic strain for the SiBAR. (a) modal displacement from FEA, (b) planar displacement along $A A^{\prime}$ ' (measured and simulated), (c) strain profile from FEA, and (d) photoelastic strain measurement along BB' (measured and simulated). Same test conditions as in Fig. 4. Simulation deformations and color scales are exaggerated for visual effect. Data in (b) and (d) are normalized to show the qualitative fit.

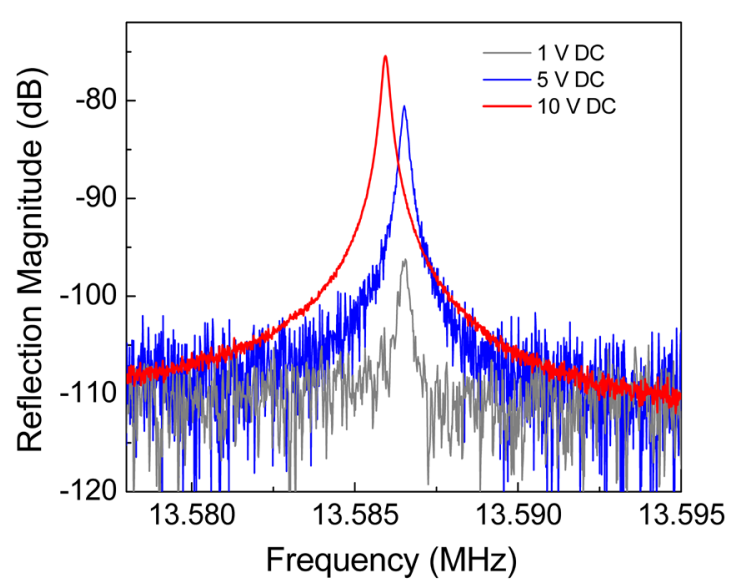

Figure 7: Knife-edge measurements as a function of DC bias. Unlike the electrical data, optical measurements have high SNR even at very low DC bias. Furthermore, optical readout is limited only by the noise in the optical path and not by device and wafer parasitics. Same test conditions as in Fig. 4.

\section{Actuation at low drive levels}

The optical techniques discussed above have far higher sensitivity to displacement and strain than the electrical measurements for the tested resonator. Within the range of $10 \mathrm{MHz}$ to $100 \mathrm{MHz}$, motion can be detected at a DC bias as low as $1 \mathrm{~V}$. An SNR of at least $15 \mathrm{~dB}$ was achieved throughout our measurements (Fig.7). The low drive voltage reduces the likelihood of inducing nonlinearities and heating, and does not require signal amplification electronics. The optical methods also make it possible to investigate thermomechanical noise in resonators and RF self-actuation. Lower RF power does not change the response magnitude, but does make the measurement noisier.

\section{Higher Modes and Frequency Limits}

The analysis above is presented for a $13.6 \mathrm{MHz}$ SiBAR, which is well within the measurement limits of the presented system. The current configuration is bandwidth limited by the photodetector (200 MHz bandwidth). SiBARs with fundamental resonance frequencies up to $120 \mathrm{MHz}$ have been measured with $\mathrm{SNR} \approx 30 \mathrm{~dB}$ and fundamental frequencies up to $220 \mathrm{MHz}$ have been detected. Higher harmonic resonance modes can also be measured with ease. Figure 8 shows the first four odd-numbered in-plane vibration modes for a SiBAR with a fundamental frequency of $28 \mathrm{MHz}$. The data shown here was measured using the knife-edge technique. We expect that the true bandwidth limits of the measurement system will be set by a combination of the decreasing displacement and strain magnitudes and increasing detector noise as the operating frequency increases. Ongoing and future experiments involve detailed system and device characterization using photodetectors operating up to $9 \mathrm{GHz}$, and will be presented elsewhere.

\section{Signal Magnitude and Quality Factor}

The magnitude of the reflected signal is a direct indicator of the displacement or strain. As such it varies over the surface of the resonator, enabling mapping of these quantities and a reconstruction of the dynamics of the resonators. For the presented SiBAR, which is fairly large, the displacement signal is stronger and less noisy than the strain signal. For smaller SiBARs, with higher frequencies and lower absolute displacements, we expect that the strain signal will be stronger than the displacement signal. 



Figure 8: Fundamental width-extensional resonance and the first three odd-numbered higher modes for a SiBAR with a width of $150 \mu \mathrm{m}$. All modes are measured using the knife-edge technique. The resonator was actuated with $10 \mathrm{~mW} R F$ power and $10 \mathrm{VDC}$ bias in air. The bandwidth of the photodetector prevents measurement of modes higher than $200 \mathrm{MHz}$.

Both measurements provide the same quality factor $(Q)$ values, at any point on the resonator, within the experimental error. This $Q$ is the direct mechanical $Q$ of the $\mathrm{SiBAR}$, and is an indicator of the intrinsic and design dependent mechanical dissipation processes (i.e., phonon loss, electron loss, thermoelastic damping, viscous damping, and tether loss). As such, these methods provide a more accurate way to measure and isolate the various dissipation mechanisms than using the 'loaded' $Q$ values found in electrical measurement techniques. For the current SiBAR, viscous damping (due to etch holes and the transduction gaps) limits the $Q$ to $\approx 10,000$ in air, and thermoelastic damping, which is exacerbated due to the etch holes, limits the $Q$ to $\approx 66,000$ in vacuum [8]. It is expected that higher frequency designs, without etch holes and operated in vacuum, will allow us to mitigate these dissipation mechanisms. The $Q$ of resonators with higher frequencies is expected to be limited by tether loss and phonon loss. The presented optical techniques will be used to study these loss mechanisms in future work.

\section{Convolved Strain and Displacement Measurements}

It is important to note that knife-edge and photoelastic measurements can be convolved when there is high strain along an edge, such as the edges of an etch hole. The strain at the leading edges of the SiBAR approaches zero so the presented knife-edge measurements were not influenced by the photoelastic effect. This was verified by measured data at points just inwards of the free edge that have near-zero photoelastic response. Conversely, at any 'solid' surface on the resonator there is no knife-edge signal, and any response is purely photoelastic. The deconvolution of the two signals based on analytical models for optical reflection will be addressed in future work.

\section{CONCLUSION}

Two complementary optical reflection measurement techniques for motion metrology of high frequency in-plane MEMS resonators were presented. The knife-edge technique can be used to measure planar displacement on any device edge with optical contrast, while the photoelastic measurement technique can be used on any clean solid material that has reasonable photoelastic coupling. Both techniques provide high sensitivity and resolution as compared to contemporary electrical methods, and are simpler to set up and use than other solutions such as 3D laser vibrometry. These techniques can be used to reconstruct the vibration mode shapes of resonators, allowing MEMS designers to better understand and optimize their designs for improved performance. These techniques also provide a more sensitive way to measure the mechanical dissipation in high frequency resonators, and can provide a path to a better experimental understanding of dissipation, even making it possible to separate and isolate different underlying dissipation mechanisms. The initial set of experiments with SiBARs indicates that there are differences between the measured and simulated mode shapes that cannot be easily explained. The possible causes for these differences and their effect on the performance of the resonator are topics of ongoing and future investigation.

\section{REFERENCES}

[1] S. Pourkamali, A. Hashimura, R. Abdolvand, G. K. Ho, A. Erbil, and F. Ayazi, "High-Q single crystal silicon HARPSS capacitive beam resonators with self-aligned sub-100-nm transduction gaps," Journal of Microelectromechanical Systems, vol. 12, pp. 487-496, Aug 2003.

[2] G. Piazza, P. J. Stephanou, and A. P. Pisano, "Piezoelectric aluminum nitride vibrating contour-mode MEMS resonators," IEEE Journal of Microelectromechanical Systems, vol. 15, pp. 1406-1418, Dec 2006.

[3] B. Gibson, K. Qalandar, K. Turner, C. Cassella, and G. Piazza, "Analysis of the impact of release area on the quality factor of contour-mode resonators by laser Doppler vibrometry," in Frequency Control Symposium \& the European Frequency and Time Forum (FCS), 2015 Joint Conference of the IEEE International, 2015, pp. 709-712.

[4] C. Rembe, R. Kowarsch, W. Ochs, A. Dräbenstedt, M. Giesen, and M. Winter, "Optical three-dimensional vibrometer microscope with picometer-resolution in $\mathrm{x}, \mathrm{y}$, and z," Optical Engineering, vol. 53, pp. 034108-034108, 2014.

[5] L. Jaesung, L. Cheng-Syun, L. Ming-Huang, C. Chi-Hang, L. Sheng-Shian, and P. X. L. Feng, "Multimode characteristics of high-frequency CMOS-MEMS resonators," in Frequency Control Symposium (FCS), 2014 IEEE International, 2014, pp. 1-3.

[6] D. Karabacak, T. Kouh, C. C. Huang, and K. L. Ekinci, "Optical knife-edge technique for nanomechanical displacement detection," Applied Physics Letters, vol. 88, p. 193122, 2006.

[7] L. Shao, M. Zhang, A. Banerjee, P. K. Bhattacharya, and K. P. Pipe, "Electrically driven nanoscale acoustic source based on a two-dimensional electron gas," Applied Physics Letters, vol. 103, p. 083102, 2013.

[8] C. Tu and J. E.-Y. Lee, "Increased dissipation from distributed etch holes in a lateral breathing mode silicon micromechanical resonator," Applied Physics Letters, vol. 101, p. 023504, 2012.

\section{ACKNOWLEDGMENTS}

Research was performed in part in the NIST Center for Nanoscale Science and Technology Nanofab.

\section{CONTACT}

*Jason J. Gorman, tel: +1-301-975-3446; gorman@nist.gov 